\title{
Xenon Gas Inhibits the Enzyme Furin In Vitro
}

David Beihl, MD, MBA, FCP

Helping Hand Labs Corporation

Correspondence to: david@getxenon.com

Abstract:

Aims:

Certain cancers, pathogenic infections, and other diseases are facilitated by the host enzyme furin, a calcium-dependent serine protease that is the most prominent member of the family of proprotein convertases. Furin and the other proprotein convertases modify certain other proteins to change them from their inactive to active forms. Previous attempts to find an effective, non-toxic furin inhibitor to treat diseases facilitated by furin have had only limited success, due to toxicity or large molecular size that impedes absorption of the molecule. This has placed increased importance on the development of small-molecule furin inhibitors. The object of this study was to consider the effect of the noble gas xenon as a furin inhibitor. 


\section{Methods:}

This study uses a fluorometric furin inhibition assay to compare the enzymatic activity of recombinant human furin after exposure to $99.999 \%$ xenon gas, compared to the enzymatic activity of untreated recombinant human furin.

\section{Results:}

Xenon exposure was found to decrease the in vitro enzymatic activity of recombinant human furin by $96-99 \%$. Fluorescence measurements were taken for 24 hours and the enzyme inhibition persisted for the duration of the experiment. Further studies will be necessary to better characterize the exact duration of this inhibition.

\section{Conclusions:}

These findings appear to be the first to report xenon as a furin inhibitor. The observed inhibition continued throughout the duration of the experiment. The effectiveness of xenon as a furin inhibitor, its favorable side effect profile, and its long history of safe use as an anesthetic, when used under direct medical supervision, make it a promising treatment for diseases facilitated by furin or its substrates. Further studies in cell culture or clinical trials may expand its clinical role for such diseases. 


\section{Introduction:}

The proprotein convertases are a group of enzymes, found in humans and other organisms, whose primary known function is to activate other proteins. In humans, there are presently nine recognized proprotein convertases. Each enzyme of this group operates by proteolytic cleavage of another protein's inactive form (the proprotein), after which the protein's activity is changed (usually increased). In some cases, the portion which is removed is also known to have biologic function. The proprotein convertases are generally felt to have overlapping, related but distinct functions from each other.

Viruses and bacteria are common causes of pathogenic infections in humans and other organisms. Many viruses rely on the proprotein convertases produced by their host to initiate and spread infection ${ }^{1,2}$. Certain bacteria also rely on these proprotein convertases, usually to activate bacterial toxins which cause disease. In addition, a number of cancers, including malignant glioma ${ }^{3}$, astrocytoma ${ }^{4}$, cervical cancer ${ }^{5}$, and at least one colon cancer cell line ${ }^{6}$, also rely on furin or its substrates for malignant growth and/or metastasis. Therefore, medications that inhibit certain proprotein convertases might prevent or treat such diseases. However, the most commonly used proprotein convertase inhibitors have a narrow therapeutic index, which means the dose that inhibits the enzyme is similar to the dose that causes toxicity in the patient. One example of this is dec-RVKR-cmk. Others are complex biological products that can be difficult to synthesize or expensive to produce, or too large in size to enter cells or be absorbed effectively by the body or delivered to the affected organ or system. One example of this is alpha-1-antitrypsin-Portland 7,8 , a modified form of alpha-1-antitrypsin (which is about $44 \mathrm{kd}$ in size). In addition, the efficacy and toxicity of many of these 
substances are often unknown, and information about the long-term side effects of their use is scarce if not absent. Thus, there is a clear and unmet need to develop effective small-molecule proprotein convertase inhibitors that can better prevent or treat bacterial, viral, or neoplastic diseases that are facilitated by proprotein convertase activity. The following experiment was conducted to evaluate the effectiveness of the noble gas xenon as a furin inhibitor.

\section{Results:}

This experiment compared the activity of recombinant human furin with and without exposure to xenon gas, using a microplate assay. The measured enzyme activity for the control and test samples is presented in Figure 1. For clarity, the 4 upper lines in the graph which rise and curve downward over the 24 hour period of measurement, represent the activity of furin in the control wells, which were not exposed to xenon. The bottom 4 flat lines, which overlap one another at the bottom of the graph, represent the minimal enzyme activity after inhibition by xenon gas.

The findings demonstrate that xenon caused a 96-99\% inhibition of furin activity; this inhibition persisted for the 24 hours during which the measurement took place. It should be noted, however, that estimates of the duration of this inhibition are not certain, because of apparent enzymatic degradation and substrate degeneration in the control wells late in the experiment, which can be seen in the graph. Test well 5 , which was exposed to xenon, only had a $96 \%$ inhibition of furin, compared to the $99 \%$ inhibition in the other three wells. This variation can be seen more clearly in Figure 2, which 
compares enzymatic activity on a logarithmic scale. The cause of this variation may be due to decreased exposure to xenon for the furin in that well secondary to uneven mixing, or perhaps to another unknown cause.

A representative subset of the graphed data is also presented in tabular form in Table 1. The numbers represent the amount of fluorescence measured in each well every five minutes. The fluorescence tends to increase in the control wells over time because the uninhibited enzyme is actively cleaving the fluorogenic substrate, which continues to fluoresce after it has been cleaved and accumulates over the duration of the experiment, until it begins to degenerate. In contrast, the wells exposed to xenon exhibit minimal to no meaningful enzymatic activity and did not increase in fluorescence; the furin in these test wells was effectively inhibited by xenon throughout the experiment. 


\section{Figure 1}

\section{Furin Activity $\left(\mathrm{s}^{-1}\right)$ With and Without Xenon Exposure vs Time}

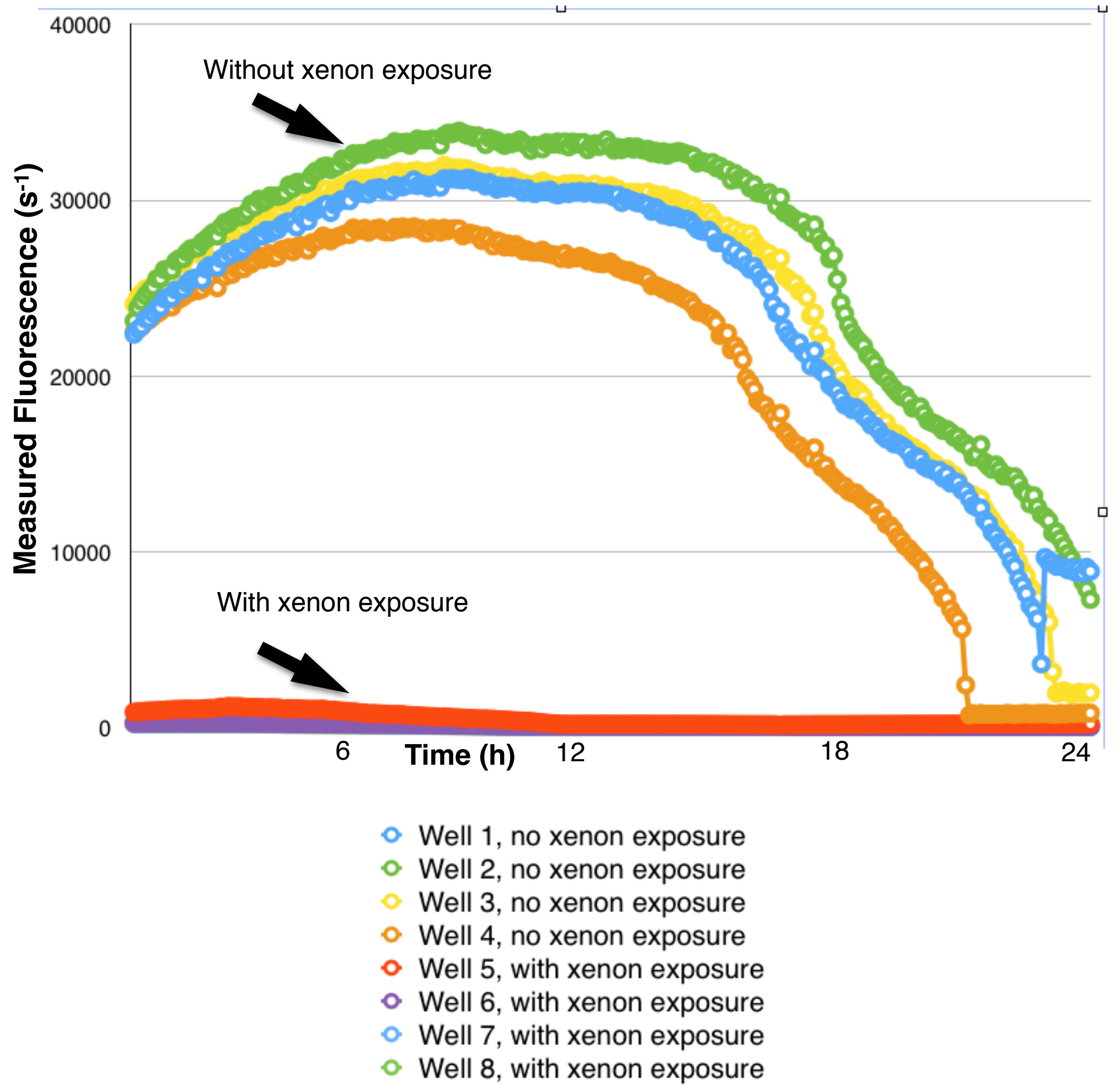




\section{Figure 2}

\section{$\underline{\log }_{10}$ of Measured Fluorescence $\left(\mathrm{s}^{-1}\right)$ vs Time}

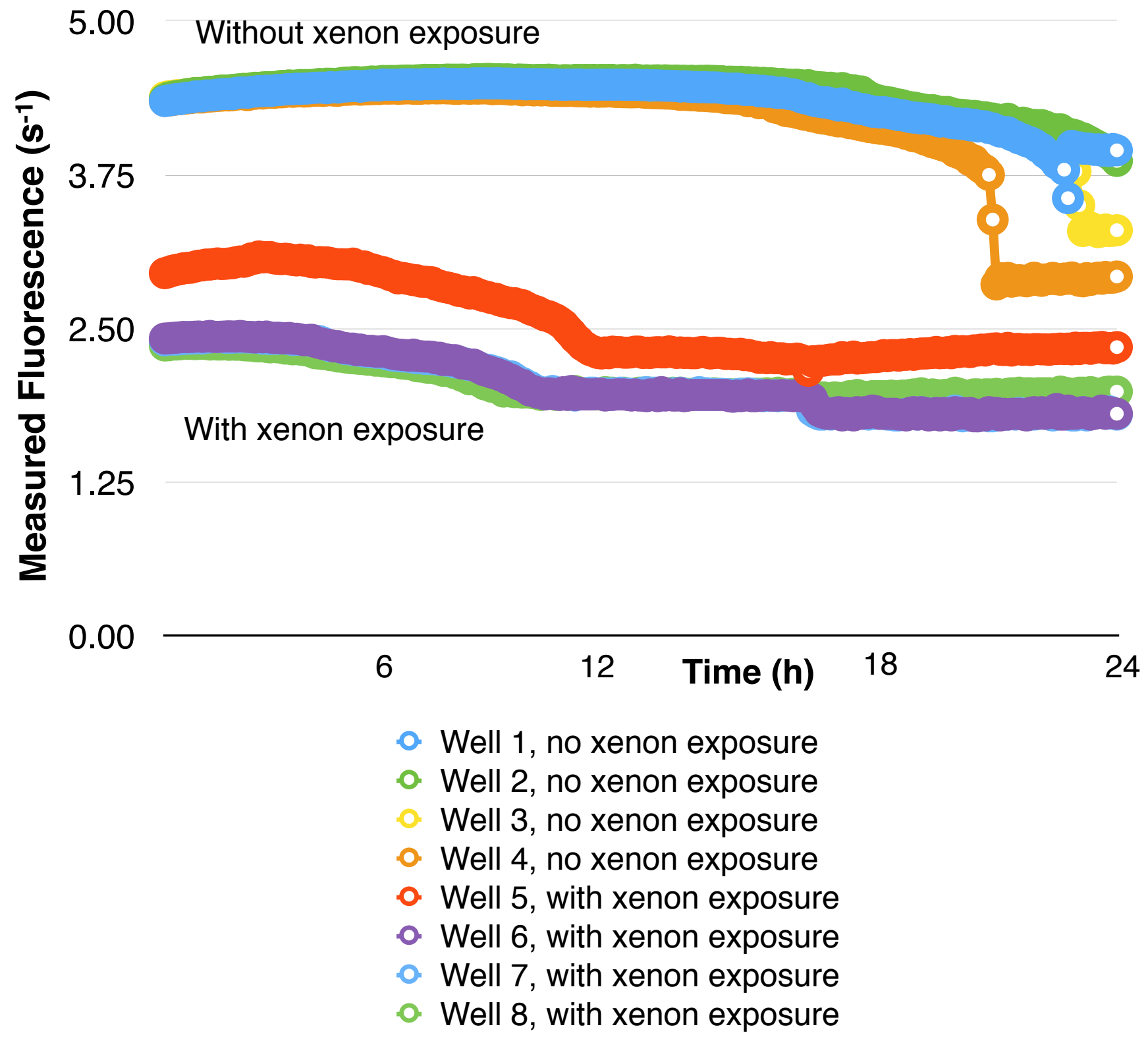




\section{Table 1}

\section{Furin Activity $\left(\mathrm{s}^{-1}\right)$ Measured at 5 Minute Intervals With and}

\section{Without Xenon Exposure}

\begin{tabular}{|c|c|c|c|c|c|c|c|c|c|c|}
\hline $\begin{array}{l}\text { Well 1- } \\
\text { No Xe }\end{array}$ & 22389 & 22662 & 22838 & 23258 & 23248 & 23589 & 23840 & 23915 & 24401 & 24519 \\
\hline $\begin{array}{l}\text { Well 2- } \\
\text { No Xe }\end{array}$ & 23144 & 23894 & 24243 & 24580 & 24835 & 25079 & 25541 & 25556 & 26061 & 26090 \\
\hline $\begin{array}{l}\text { Well 3- } \\
\text { No Xe }\end{array}$ & 24090 & 24501 & 24728 & 24964 & 25019 & 25117 & 25540 & 25518 & 26039 & 25975 \\
\hline $\begin{array}{l}\text { Well 4- } \\
\text { No Xe }\end{array}$ & 22503 & 22656 & 22815 & 23072 & 23204 & 23382 & 23557 & 23687 & 23973 & 23954 \\
\hline $\begin{array}{l}\text { Well 5- } \\
\text { with Xe }\end{array}$ & 891 & 909 & 932 & 947 & 959 & 977 & 984 & 988 & 1007 & 1026 \\
\hline $\begin{array}{l}\text { Well 6- } \\
\text { with Xe }\end{array}$ & 262 & 263 & 265 & 266 & 268 & 270 & 269 & 270 & 271 & 272 \\
\hline $\begin{array}{l}\text { Well 7- } \\
\text { with Xe }\end{array}$ & 253 & 257 & 257 & 257 & 258 & 259 & 264 & 261 & 262 & 264 \\
\hline $\begin{array}{l}\text { Well 8- } \\
\text { with Xe }\end{array}$ & 230 & 231 & 234 & 234 & 235 & 241 & 237 & 237 & 236 & 237 \\
\hline $\begin{array}{l}\text { Time } \\
(\min )\end{array}$ & 0 & 5 & 10 & 15 & 20 & 25 & 30 & 35 & 40 & 45 \\
\hline
\end{tabular}




\section{Discussion:}

This study appears to be the first to report xenon as a furin inhibitor. The observed inhibition continued throughout the duration of the experiment. Since no enzyme activity was measured for 24 hours after xenon exposure, although the enzyme and substrate were both present, the effect of xenon lasted at least this long, although perhaps it would have lasted longer if the experiment had been continued. The effectiveness of xenon as a furin inhibitor, its favorable side-effect profile, and its long history of safe anesthetic use when used under direct medical supervision, make it a promising treatment for the viral, bacterial, neoplastic, and other diseases facilitated by furin or its substrates. Further studies using xenon in cell culture or in clinical trials may clarify its clinical role for such diseases.

\section{Materials and Methods:}

The in vitro testing in this study was performed using a standard fluorometric furin activity assay (Anaspec: Fremont, CA). This assay uses a fluorometric substrate to measure the enzymatic activity of recombinant human furin. When the enzyme is active, it breaks the substrate in such a way that a chemical compound is released that will fluoresce when exposed to light at the proper wavelength. The assay includes appropriate quantities of the reagents for microplate studies and is designed to be suitable for screening substances for furin inhibition as part of a medication discovery process. 
The instructions provided with the assay were carefully followed regarding the reconstitution of the furin enzyme, the fluorogenic substrate, and the other components of the assay. The reconstituted furin enzyme was placed in a Schlenk flask. Schlenk flasks are special flasks that have a side port for the addition or removal of gases. They are useful for studies involving a gaseous reagent.

First, the control wells on the microplate were filled from the untreated enzyme solution in the Schlenk flask. Then the air was then gently removed from the Schlenk flask with a manual vacuum pump. None of the liquid containing the enzyme was visibly removed when the air was removed. The Schlenk flask was subsequently attached to a source of research-grade 99.999\% xenon gas (Advanced Specialty Gases: Reno, NV), and xenon gas was added to a pressure of approximately 10 psi above atmospheric pressure.

After filling the flask to this pressure, the xenon gas was disconnected, and the xenon-exposed furin enzyme was then micropipetted into the test wells of the microplate, where its substrate had already been placed. Placing the fluorogenic furin substrate in the microplate wells in advance minimized the time delay between the activity of the control wells and of the test wells.

Before the last set of test wells were filled, the Schlenk flask was grasped manually and briefly moved gently in a circular manner, with a radius of about $15 \mathrm{~cm}$, to allow for improved exposure of the enzyme to xenon. Since the dissolution of xenon in water is an endergonic process, it is poorly soluble in water ${ }^{9}$; this gentle manual mixing facilitated the ability of xenon to contact the furin enzyme, without causing enzyme denaturation. The black, 96-well, non-binding surface microplate was subsequently 
placed in our Tecan Infinite F200 microplate reader (Tecan Group Ltd: Männedorf, Switzerland) and underwent automatic fluorescence measurements every 5 minutes for 24 hours. The findings from these final set of test wells are presented in Figure 1 and Figure 2. An initial subset of these findings is also presented in Table 1.

\section{Acknowledgements:}

The work of those who developed and produced the Anaspec furin activity assay made this study much easier than it otherwise would have been. Their service is gratefully appreciated. The gracious assistance of the staff at Advanced Specialty Gases is also recognized.

Dr. Beihl performed the research and authored this entire paper. He holds numerous patents pending on pharmacological compositions and methods of using noble gases to inhibit proprotein convertases, including methods of their clinical use in the treatment of cancers, certain viral and bacterial infections, degenerative diseases, and vascular diseases, as well as autoimmune and other diseases. No governmental, institutional, or other external funding was received to perform this research. 


\section{References:}

1 https://www.ncbi.nlm.nih.gov/pmc/articles/PMC16021/

2 https://pubmed.ncbi.nlm.nih.gov/32362314/

3 https://pubmed.ncbi.nlm.nih.gov/11390472/

${ }^{4}$ https://pubmed.ncbi.nlm.nih.gov/12060611/

${ }^{5}$ https://pubmed.ncbi.nlm.nih.gov/25818795/

6 https://pubmed.ncbi.nlm.nih.gov/11402025/

7 https://pubmed.ncbi.nlm.nih.gov/11062061/

8 https://pubmed.ncbi.nlm.nih.gov/10681468/

${ }^{9}$ https://pubmed.ncbi.nlm.nih.gov/14499905 